\title{
Antigen Presentation After Stroke
}

\author{
Francesc Miró-Mur $^{1} \cdot$ Xabier Urra $^{1,2} \cdot$ Mattia Gallizioli $^{3} \cdot$ Angel Chamorro $^{1,2}$ • \\ Anna M. Planas ${ }^{1,3}$
}

\begin{abstract}
Stroke induces a local inflammatory reaction and a plethora of innate immune responses in the brain where antigenpresenting cells become prominent. However, to date, it is still unclear whether antigen presentation is relevant to the neuropathological and functional outcome of stroke. Stroke does not trigger overt autoimmune reactions, but neural antigens have been found in lymphoid tissues of patient with stroke and it is unknown whether they promote tolerance or immune reactions that under certain conditions might contribute to the functional worsening observed in some patients. Autoantibodies to neural molecules have also been reported in patients with stroke, but the subclass of antibodies is important for their function, and the contribution of such findings to stroke outcome is not yet clear. Notably, stroke induces immunodepression highlighted by a transient lymphopenia, lymphoid organ atrophy, and monocyte deactivation. While these effects might reduce the chances of autoreactivity, they increase the risk of infection in patients with stroke and most frequently in those with severe stroke. Therefore any potential brain protective effect of strokeinduced immunodepression by attenuating or preventing lymphocyte-mediated brain damage is confounded by stroke severity and an increased incidence of infections. Systemic inflammation due to a number of comorbidities that are frequent in patients with stroke is also associated to a poor outcome.
\end{abstract}

Anna M. Planas

anna.planas@iibb.csic.es

1 August Pi i Sunyer Biomedical Research Institute (IDIBAPS), Barcelona, Spain

2 Functional Unit of Cerebrovascular Diseases, Hospital Clínic, Barcelona, Spain

3 Department of Brain Ischemia and Neurodegeneration, Institut d'Investigacions Biomèdiques de Barcelona (IIBB), Consejo Superior de Investigaciones Científicas (CSIC), Barcelona, Spain
Herein, we review some relevant findings regarding the identification of neural antigens in stroke and discuss their potential contribution to the functional outcome of stroke.

Keywords Brain antigens · Inflammation · Innate immunity · Adaptive immunity $\cdot$ Stroke

\section{Introduction}

Neuroinflammation is a hallmark of many acute and chronic brain diseases. It comprises an enhanced release of several cytokines in the brain tissue, the reaction of innate immune cells resident in the brain parenchyma, i.e., the microglia, and often involves the infiltration of myeloid cells, and adaptive immune cells, i.e., lymphocytes. Under physiological conditions, immune cells surveil the brain at the perivascular spaces, the leptomeninges, and the choroid plexus, and, from these strategic locations, they can sample the brain environment [1]. Following acute stroke a variety of immune cells can access the brain parenchyma in an orchestraded manner [2, 3]. Acute brain damage generates a genuine inflammatory reaction triggered by necrotic neuronal cells that leak their content to the extracellular space and trigger strong innate immune responses involving resident microglia and infiltrating leukocytes [4, 5]. Notably, antigen-presenting cells (APC) accumulate in the brain parenchyma during the days that follow experimental stroke [6-10]. These cells express major histocompatibility complex (MHC) class II and co-stimulatory molecules, as well as markers of conventional dendritic cells (DCs). The presence of cells expressing MHC class II in the brain has also been reported in human stroke [11]. T cells are known to have detrimental effects in stroke by promoting leukocyte adherence to the brain vasculature [12] and triggering thromboinflammation [13] in animals. The presence of T cells and $\gamma \delta$ lymphocytes was also reported 
in mouse and human brain tissue $24 \mathrm{~h}$ after stroke [14]. These effects are rapid and lack antigen specificity. In addition to this early nonspecific effect of T cells, is there room for any antigenmediated responses in acute stroke? Several lines of experimental evidence suggest that this might be the case [15-21], but further study is needed to confirm this possibility and dissect its potential contribution to the brain tissue fate and the potential relevance to human stroke. Indeed, our group described the presence of several brain antigens in the draining lymphoid tissue of patients with acute stroke that was correlated with the functional outcome of the patients at follow-up [22]. Recently, a study of brain ischemia in mice reported increased autoreactive $\mathrm{T}$ and $\mathrm{B}$ cells to human neural antigens [20].

Besides the vascular route after leakage of the blood-brain barrier (BBB) [23], brain antigens might reach the cervical lymph nodes after stroke following several anatomical pathways identified in animals and humans. These routes allow the drainage of interstitial fluid and solutes out of the brain parenchyma through the glymphatic system [24-28]. Brain fluid and macromolecules can eventually reach the cervical lymph nodes along extracranial nerves through the cribiform plate [29-31], and through the recently described lymphatic vessels in the dura matter of the mice $[32,33]$. Thus, APCs would capture neural antigens in the lymph nodes. Alternatively, APCs could take up antigen in the brain and then travel to the lymph nodes [34]. Expectedly, the presence of immature DCs presenting brain antigens in the lymph nodes would induce tolerance by causing anergy of $\mathrm{T}$ lymphocytes or by expanding regulatory T cells (Tregs) [35]. Yet, antigen presentation and the associated T-cell reaction depends on the local microenvironment, and the presence of co-stimulatory molecules and Tregs. Therefore, it is possible that effector T cells could be generated under certain circumstances that are described following acute stroke, i.e., inflammation or infection, thus breaking peripheral tolerance and generating autoimmune responses.

Stroke induces depression of peripheral immune responses through neurotransmitters released by the sympathetic nervous system, as described in animals and humans [5, 36]. The interaction between the central nervous system (CNS) and the immune system is complex as shown by the recent observation that Treg cells in the gut regulate the infiltration of $\gamma \delta \mathrm{T}$ cells in the ischemic mouse brain tissue [37]. This crosstalk could be an adaptive response to dampen brain damage and limit harmful immune responses to brain-derived antigens. Yet, a critical and unresolved question is whether presentation of CNS antigens actually drives an immune response following brain ischemia. The identification of autoantibodies in patients with stroke could be an indication of autoimmunity, but this finding requires a cautious interpretation as not all antibodies have similar functions. Classically, brain autoimmunity is mediated by $\operatorname{IgG}$ autoantibodies [38], which are rarely found in acute stroke. In contrast, the most frequently reported autoantibodies are of the IgM or IgA subclass [39].
As discussed below, the clinical impact of these autoantibodies also remains to be established.

\section{Innate Versus Adaptive Immunity in Stroke}

Activation of innate immune cells in the damaged brain a few hours after an ischemic event participates in infarct growth by triggering inflammatory signaling [4, 40, 41]. Brain cell death elicits the production of danger signals that activate innate immune cells, in compliance with the danger model in immunity [42]. Adenosine triphosphate, heat-shock protein, and high mobility group box-1 are amongst the molecules described as danger-associated molecular patterns released in brain ischemia. Binding of these molecules to pattern recognition receptors, such as Toll-like receptors (TLRs), displayed by microglial cells and infiltrated innate immune cells, participates in the inflammatory response. An essential function of innate immunity is to provide the informational input for adaptive immunity. APCs play a critical role in initiating T-cell responses by taking up protein antigens in tissues, processing them into small peptides and then displaying them on their surface, physically associated with MHC class I to activate CD8 T cells, or with MHC class II to educate CD4 T cells. Hence, danger-associated molecular patterns could be acting as co-stimulatory molecules furnishing the required signals to activate APCs fully.

DCs are the most potent APCs and provide a critical link between innate and adaptive immunity. While microglial cells were believed to be the main APC population in the CNS, studies in experimental autoimmune encephalomyelitis (EAE) established that a discrete population of vesselassociated $\mathrm{CD} 11 \mathrm{c}^{+}$cells is responsible for antigen presentation to myelin-reactive T cells [43]. Juxtavascular APCs are also described in healthy rodent brains where they project cellular processes into the glia limitans and could present brain parenchyma-antigens to circulating T cells [44]. In addition, populations of interferon- $\gamma$ responder mouse brain resident DCs may behave as effective APCs [45]. Interestingly, these cells resembled activated microglia, expressed Ibal and $\mathrm{CD} 11 \mathrm{~b}$ markers, and had the ability to migrate and concentrate in the choroid plexus, a critical region for the entry of peripheral T cell into noninflamed CNS [46]. Additional evidence suggests that under physiological conditions the population of Iba $1^{+} \mathrm{CD} 11 \mathrm{~b}^{+} \mathrm{CD} 11 \mathrm{c}^{+}$cells resident in the brain corresponds to a subset of microglial cells [47]. Further study is needed to clarify fully the identity and function of these DC-like brain cells. Following stroke, microglia, infiltrated macrophagesmonocytes, and DCs express MHC class II, and costimulatory molecules, thus they can act as APCs in the ischemic brain parenchyma $[2,8,10]$. However, antigen processing differs between macrophages and DCs, as the latter regulate lysosomal acidification better than the former in order to preserve peptides for T-cell recognition [48]. Priming of T cells 
mainly takes place in the draining lymph nodes where migratory DCs carrying the antigens get in close proximity to naïve $\mathrm{T}$ cells. In photothrombotic stroke models an increase in migratory $\mathrm{CD} 11 \mathrm{c}^{+} \mathrm{CD} 103^{+}$cells has been reported [9]. Whether these cells will reach the draining lymph nodes to present antigen to T cells remains elusive, but our study of tonsils from patients with stroke, i.e., lymphoid tissue located within the draining pathways of the CNS, showed neural antigens on migratory $\mathrm{BDCA}^{+}$cells [22].

The way DCs are activated to present antigens could determine the initiation of immune reactions or the induction of tolerance [49]. Immmature DCs are mainly responsible for antigen uptake and migration to secondary lymphoid tissues. Signaling through TLRs and inflammatory molecules induces DC maturation, a process required for antigen processing and antigen presentation [50]. Lymphocytic activation represents a key step of the adaptive immune response. The molecular requirements for full T-cell priming involve two signaling events, recognition by the T cell receptor (TCR) of antigen presented on a major histocompatibility complex (pMHC) on the surface of an APC, as well as signaling from co-stimulatory and adhesion molecules, and T-cell polarizing factors, all of them coming from the same APC [51, 52]. In addition to these molecular interactions, the physical forces created by $\mathrm{T}$ cell receptorpMHC interaction at the immune synapse also determine the nature of T-cell activation [53, 54]. Activated T cells will then migrate to the inflamed tissue where they need to re-encounter their cognate antigen in the context of MHC-bearing APCs to perform their effector function.

\section{Neural Antigens in Stroke}

Neural antigens such as myelin basic protein (MBP), neuronspecific enolase, and S100 $\beta$ can be found in the serum of patients with stroke, and their concentrations are correlated with the severity and volume of infarction [55]. Several lines of evidence suggest that stroke might trigger neural antigen presentation. However, this possibility is not fully demonstrated and many questions must be resolved. How CNS antigens are delivered to APCs and whether antigen is captured and presented by the same APCs to prime T cells in tissue-draining lymph nodes are elusive. While DCs can transport antigens to the lymph nodes, resident microglia are unlikely to traffick out of the CNS. An increased presence of brain-derived antigens in migrating DCs and macrophages at the draining lymphoid tissue was described in rodent models of EAE, experimental stroke, patients with multiple sclerosis (MS) [56], and patients with stroke [22]. Besides trafficking of migratory DCs carrying neural antigens, soluble brain peptides could access the lymph nodes (see above) and be taken up by APCs locally. Interestingly, microscopic observations suggested that brain-antigen immunoreactive macrophages contact lymphoid resident
DCs surrounded by some activated T cells [22]. These observations raise the possibility of cargo exchange between different APCs enabling the resident professional APCs to present neural antigens. The presence of activated $\mathrm{T}$ cells near the antigenbearing APCs might ensue $\mathrm{T}$ cell response to brain antigens. Ortega et al. [20] further explored this notion in a mouse model of experimental stroke and found that lymph node T and B cells from ischemic mice showed autoreactivity to human CNS antigens when re-stimulated in vitro. Therefore, adaptive immune responses against CNS antigens could be initiated in the periphery and subsequently propagated to the CNS by circulating primed T cells, which would be re-stimulated by antigens within the CNS [57]. Myeloid cells found in the meninges and choroid plexus, as well as perivascular macrophages, could act as functional APCs that could re-activate cerebrospinal fluid (CSF) circulating primed $\mathrm{T}$ cells [46]. Functional characterization of the T-cell immune response against brain-derived antigen is needed because the APC challenge to T cells can elicit autoimmune response to self-antigens or a tolerogenic response, depending on the co-stimulatory signaling and local microenvironment. Furthermore, it is unknown whether any potential antigen-T-cell autoreactivity influences stroke outcome, but several studies have described an increased immunoreactivity to neuronal protein microtubule-associated protein (MAP)-2 in lymphoid tissue that was associated with smaller infarcts [20, 22]. Interestingly, dampening MAP-2 degradation minimizes ischemic injury in the cerebral cortex [58]. MAP-2 autoimmune response could either preserve the integrity or promote the recovery of neurons at risk of dying in the injured ischemic tissue [20, 59]. In contrast, antigen responses to myelin proteins have been associated to autoimmunity and exacerbation of the brain injury in experimental stroke [60]. Accordingly, immunoreactivity to myelin peptides in the human draining lymph nodes was associated with greater infarcts and worse outcome [22].

The timing of any autoimmune response is also critical. Whereas some authors reported $\mathrm{CD} 8^{+}$and $\mathrm{CD} 4^{+} \mathrm{T}$-cell recruitment within $24 \mathrm{~h}$ in ischemic [61] or hemorrhagic [62] stroke models, other experimental studies showed homing of T lymphocytes [2] or Tregs [63] 4 days after stroke, or later [64]. Also, the presence of $\mathrm{T}$ lymphocytes in human brain tissue has been reported 3-4 days after stroke [11]. As autoreactivity of $\mathrm{T}$ cells in the periphery was not observed before 4 days [20], the assumption of an antigen-dependent T-cell autoimmune response in the injured brain fits with a delayed entry of lymphocytes into the ischemic tissue. This finding does not exclude the possibility of priming and licensing of T lymphocytes by APCs inside the injured brain parenchyma. However, in EAE, MBP-reactive lymphocytes must be transferred into the peripheral circulation to induce clinical symptoms. In the periphery, these encephalitogenic T cells first enter secondary lymphoid tissues, where the expression of activation markers and chemokine receptors is synchronized and later on they are licensed in the lungs before 
entering the CNS [65]. Potentially, antigen-dependent T-cell responses early after stroke onset could take place in the presence of memory $\mathrm{T}$ helper cells that might occur in patients with a history of transient ischemic attacks or stroke.

DCs can be tolerogenic depending on their functional and maturation status and are able to delete or silence autoreactive $\mathrm{T}$ cells and facilitate the development of Tregs [66, 67]. A pathway for DCs to traffic via the rostral migratory stream from the brain to the cervical LN, where they present antigen to $\mathrm{T}$ cells for inducing immune tolerance was observed in a mouse model of MS [68]. The neuroprotective effect of these DC-mediated Tregs could be negatively affected by local sequestration induced by the immunomodulatory drug fingolimod [68]. Antigen can be processed and presented in a context that induces Tregs, which exert an immunomodulatory effect in response to antigen exposure and prevent activation of lymphocytes [69]. In experimental stroke, Tregs proliferate and accumulate in the ischemic zone during the first month [64], and increase in secondary lymphoid organs, suggesting participation in systemic immunodepression [70]. Nonetheless, the role of Tregs in stroke outcome is controversial. While Treg conferred neuroprotection in certain experimental stroke models [63], they did not seem to affect the neurological outcome in other studies [64]. Furthermore, some studies highlighted the deleterious function of Tregs in acute stroke by inducing dysfunction of the cerebral microvasculature [71], also after Treg boosting with CD28 superagonist [72]. However in other studies, a neuroprotective role of Tregs was observed when these cells were expanded with CD28 superagonist [73], after inhibiting histone deacetylases [74], or after Treg adoptive transfer [74-76].

\section{Autoantibodies}

Antibodies against brain antigens have been found in CSF of patients with stroke [77, 78], suggesting that the release of brain antigens, especially when BBB dysfunction occurs, may be followed by intrathecal B-cell production. The presence of IgG, IgM, and IgA subtypes of autoantibodies has been described [78]. Autoantibodies have also been reported in the serum of patients with stroke, including antineurofilament antibodies [79] and antibodies against the N-NR2A/2B subtype of the $N$-methyl-D-aspartate receptor (NMDAR) [80, 81]. Nevertheless, the presence of some autoantibodies has been described in many different pathological conditions, and also in healthy subjects $[82,83]$, but their clinical significance is not clear. It is possible that the loss of BBB integrity was critical to enable pathogenic effects of circulating autoantibodies. NMDAR1 autoantibodies seem to be beneficial or detrimental, depending on the BBB integrity before stroke. Using APOE4 carrier status as an indicator of a preexisting leaky BBB, Zerche et al. [39] observed greater infarct growth in patients with BBB disruption and autoantibodies against NMDAR, whereas patients without previous BBB disruption that had autoantibodies showed the smallest infarct growth. Autoantibodies may also have a role in the development of poststroke cognitive impairment. In mouse models, infiltration by B lymphocytes occurred weeks after cerebral ischemia, in parallel with the appearance of signs of cognitive decline [84], and IgG autoantibodies against MBP after stroke were more frequent in patients with cognitive decline than in patients without it [85].

Nonetheless, it is important to note that the immunoglobulin isotype of autoantibodies has relevant pathogenic implications [86]. The majority of the autoantibodies against extracellular epitopes like NMDAR1, myelin oligodendrocyte glycoprotein, and contactin-associated protein-like 2 detected in healthy subjects or in patients with different neurological diseases, including stroke, are IgA- or IgM-positive, with very few IgG-positive samples [83]. In contrast, only IgG autoantibodies have been proven to be pathogenic in antibodymediated neurological autoimmune diseases, such as antiNMDAR encephalitis [87], whereas the clinical significance of the presence of IgA or IgM autoantibodies is debated [88].

\section{Systemic Inflammation}

Inflammation is a classical response to infection and injury with many beneficial effects, but inappropriate inflammation can precipitate cerebral ischemia or aggravate it. Large community studies have found associations between systemic inflammatory conditions and cardiovascular disease, including stroke [89, 90]. Inflammation is one of the main pathogenic mechanisms in atherosclerosis, and considering the interaction between the circulation and the immune system, it is not surprising that there is a tight association between atherosclerosis and systemic autoimmunity. Cholesterol accumulation in macrophages and other immune cells promotes inflammatory responses, including augmentation of TLR signaling, inflammasome activation, and the production of monocytes and neutrophils in the bone marrow and spleen [91]. Antigen-specific adaptive immune responses may also have a role in the atherogenic process [92], as suggested by studies in mouse models of atherosclerosis and mice deficient in both B cells and T cells [93, 94]. In turn, atherosclerosis may also enhance autoimmune responses in other organs, including the brain. The production of interleukin (IL)-17 and IL-17-promoting cytokines is enhanced in atherosclerosis. Macrophages and DCs produce IL- $1 \alpha$ and IL- $\beta$, while $\gamma \delta \mathrm{T}$ cells and innate lymphoid cells produce IL-17 [95]. DCs also exhibit increased expression of TLR4, IL-1R1, and caspase-1 in atherogenic conditions [96]. Systemic levels of IL-17, IL-21, and IL-23 are increased in patients with atherosclerosis [97]. These proinflammatory effects of atherosclerosis can enhance autoimmune responses in the brain. In atherogenic mice, naïve autoreactive $\mathrm{T}$ cells preferentially differentiate into Th17 cells, 
leading to exaggerated autoimmune inflammation in CNS [98]. Rheumatic diseases are also an independent risk factor for stroke [99]. The risk is higher in patients under 50 years and is most probably related to inflammatory mechanisms, because the risk of stroke is greater in inflammatory arthropaties compared with noninflammatory osteoarthritis [100]. Inflammation in rheumatic diseases is systemic, nonresolving, and often only controlled with aggressive antirheumatic drugs. The risk of stroke also seems to increase in other conditions associated with excessive inflammation such as chronic obstructive pulmonary disease in the weeks following an exacerbation [101].

The study of the effects of systemic inflammation over the fate of cerebral ischemia is challenging because many experimental studies are carried out in animals without comorbidities that do not adequately mimick the presence of systemic inflammation [102]. However, considerable evidence supports a relationship between systemic inflammation and poor outcome in patients with stroke and in experimental stroke models. Many components of the immune system are involved in the pathological processes underlying the development of atherosclerosis and its ischemic complications [103]. In patients, stroke can produce an inflammatory response known as systemic inflammatory response syndrome, which is especially frequent in patients with severe stroke. Among patients treated with thrombolysis, those who developed systemic inflammatory response syndrome were at greater risk of poor functional outcome even after adjusting for relevant prognostic confounders [104]. Experimental models of stroke have shown that various systemic inflammatory mechanisms exacerbate brain damage and worsen functional deficits. The mechanisms by which systemic inflammation exhacerbates cerebral ischemia are diverse, including reduced reperfusion after vessel recanalization [105], increased BBB injury, and edema formation [106]. Raising systemic inflammation in experimental ischemia through IL-1 $\beta$ administration, or in IL-10-deficient mice, or in mice with chronic infection that have a $\mathrm{T}$ helper 1 (Th1)-polarized immune response, has been associated to increased disruption of tight junction proteins and basal lamina collagen, increased platelet aggregation, microvascular injury, increased MMP activation, and increased mortality after experimental ischemia [102, 107, 108]. Tight junctions between endothelial cells and the epithelial cells of the blood-CSF barrier limit access to the CNS by circulating cells [109], but activated T cells can enter to the CNS in inflammatory situations because they express adhesion molecules, chemokine receptors, and integrins [110].

\section{Stroke and Infection}

Infections are frequent both before and after stroke [111], and it is possible that the timing of the infections is a key factor in modulating the immune response [112]. Both acute and chronic infections like bronchial or dental infections can promote atherosclerosis and be a precipitant of stroke both in the general population [113] and in surgical patients, who have a 3-fold risk of both arterial and venous thrombosis in case of preoperative sepsis [114]. Strokes in patients with previous infection manifest greater deficits, and increased platelet activation and platelet-leukocyte aggregation compared with patients without previous infection [115]. In young patients with stroke, previous infection increased the risk of death and disability at 3 months [116]. Thus, the presence of previous infections could facilitate the occurrence of stroke and impair functional recovery. However, stroke-associated infection (SAI) is the most frequent medical complication of stroke, with an incidence that ranges from $13 \%$ to $45 \%$ in most recent studies [117, 118], most often involving respiratory and urinary tract infections associated with significant mortality and increased costs [119].

Of general concern is that SAI may be a source of inflammation and autoimmunity as infection facilitates the maturation of APCs into potent immunostimulatory cells [120], and is involved in the development of autoimmune diseases [121, 122]. The effects of infections in cerebral ischemia have often been tested in experimental models by administration of bacterial lipopolysaccharide at the moment of cerebral ischemia. Lipopolysaccharide increased the likelihood of developing a detrimental autoimmune response to brain antigens with higher presence of B7.1 co-stimulatory molecules on APCs and greater Th1 responses to MBP [123-125]. Becker et al. [124] also showed that patients who developed an infection after stroke were more likely to show a Th1 response to MBP and glial fibrillary acidic protein, and stronger Th1 responses to MBP were associated to poor functional outcome. Nonetheless, it is possible that the induction of systemic inflammation or infection in experimental animals at the time of cerebral ischemia will mimic better the clinical scenario of infections precipitating a stroke rather than the infections occurring in the aftermath of stroke.

\section{SAI and Brain Damage}

SAI also increased the mortality rate and the risk of long-term recurrent vascular events in some studies [116, 118]. However, studies aimed at analyzing the prognostic consequences of SAI have often failed to find an independent deleterious effect of infections, suggesting that several confounders could sway [126]. The determinants of SAI are multifactorial and include the severity of neurological deficits, dysphagia that favors bronchoaspiration, and a number of systemic immune changes dubbed as stroke-induced immunodepression syndrome [5]. Immunodepression has been mainly related to the clinical severity of the stroke and to the size of the brain lesion both after experimental and clinical brain ischemia [127, 128]. This could have therapeutic implications because the immunological features of small and large infarctions may be different. 
Stroke-induced immunodepression was first described in a murine model of cerebral ischemia where overactivation of the adrenergic system caused lymphopenia, a shift from Th1 to Th2 cytokine production, and atrophy of primary and secondary lymphoid organs [36, 70]. In addition to stress hormone release, large stroke volumes could also propitiate a greater incidence of SAI as the result of an increased release of danger signals. For instance, high mobility group box-1 release from the ischemic brain induced the proliferation of bone marrow-derived suppressor cells, inhibiting the adaptive immune responses and resulting in lymphopenia and functional exhaustion of monocytes [74]. In patients with stroke, the best established features of SAI are increased levels of stress hormones and antiinflammatory cytokines like IL-10 [124-131], decreased numbers of circulating lymphocytes [129, 131, 132], and monocyte deactivation with reduced expression of human leukocyte antigen-antigen D related and reduced capacity to produce inflammatory cytokines $[129,131]$.

Immunosuppression could be a compensatory response to protect the ischemic brain from excessive inflammation, at expenses of lowering the infectious threshold [133, 134]. Most features of the stroke-induced immunodepression may favor tolerogenic immune responses. Thus, catecholamines can inhibit the antigen-presenting capability via $\beta 2$-adrenoceptors [135-137], corticosteroids inhibit the production of inflammatory cytokines in APCs and induce the development of tolerogenic APCs [138, 139], and glucocorticoid-stimulated monocytes reduce the release of interferon- $\gamma$ and IL-17 in lymphocytes, favoring the generation of Tregs [140]. IL-10 also inhibits autoimmune reactions acting on several immune cells, including APCs inducing anergic T cells that are able to suppress activation, and function of $\mathrm{T}$ cells in an antigen-specific manner [141]. In addition, other alterations in the numbers and phenotype of circulating leukocytes, such as lymphocytopenia and reduced human leukocyte antigen-antigen D related expression in monocytes, could further impair the activation of specific T-cell responses against brain antigens.

Interestingly, enhancing immunity after cerebral ischemia may prevent infections at the expense of favoring autoimmunity. The pharmacological blockade of the sympathetic nervous system and the hypothalamic-pituitary-adrenal axis in a murine model of cerebral ischemia reduced infarct volume, decreased infection rate, and increased long-term survival, but increased CNS antigen-specific Th1 responses in the brain [21]. Even if autoimmunity was not detrimental in this experimental model, these results confirm the idea that the balance of proinflammatory and anti-inflammatory mediators may be crucial in the appearance of antigen specific autoimmune responses after stroke.

The relationship between autoimmune responses and ischemic brain lesion is further complicated by the observation that myelin oligodendrocyte glycoprotein-specific autoreactive $\mathrm{CD} 4 \mathrm{~T}$ cells are required for normal learning behavior in experimental animals [142], and that, in the absence of infection, the brain self-antigen T-cell response is
Fig. 1 Stroke causes brain cell death and the release of brain antigens that can reach the lymph nodes either as solutes in the interstitial fluid or can be captured by dendritic cells (DCs) or antigen presenting cells trafficking to the lymph nodes. Antigen presentation to $\mathrm{T}$ cells under inflammatory conditions can trigger autoreactivity that can exacerbate brain damage, or can promote tolerance by regulatory $\mathrm{T}$ cells (Treg) that will dampen inflammation and exert beneficial effects

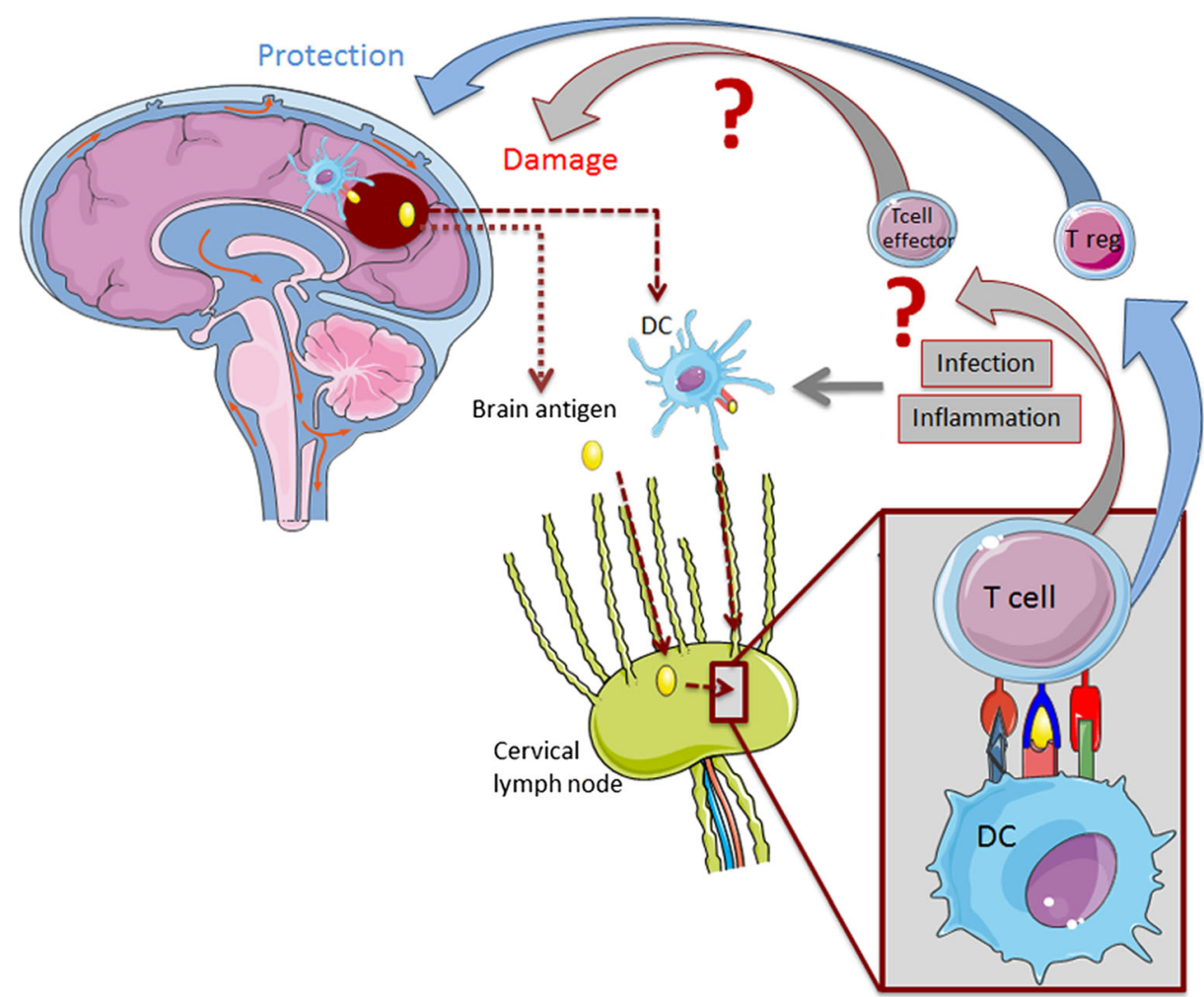


tolerogenic [143]. Some immune responses against selfantigens may induce protective autoimmunity and hence could be participating in CNS repair [144].

In theory, anti-inflammatory or immunosuppressive treatments might further increase the risk of infections in patients with severe strokes that spontaneously develop systemic immunodepression but may benefit patients with smaller ischemic lesions that are not at risk of infection. Immunomodulatory drugs that are used in patients with MS are now being tested in stroke. For instance, fingolimod, a drug that induces lymphopenia and prevents lymphocyte access to the brain, has shown beneficial effects in stroke animal models [145] and in small clinical trials in ischemic patients with stroke [146, 147]. Also, natalizumab, a drug that inhibits leukocyte infiltration, seems to exert some benefits in human stroke (ACTION trial), and a corresponding mouse anti-CD49d antibody has shown protective effects but only in 1 of 2 experimental models of brain ischemia [148]. Larger studies in patients with stroke are needed to demonstrate efficacy and safety of immunodulatory strategies. However, patient stratification might be required to identify those subpopulations that could benefit from immunomodulation.

\section{Final Remarks}

We have reviewed the current status of antigen presentation in stroke and summarize it in Fig. 1. Overall, we described that brain antigens have been detected in the draining lymphoid tissue and the blood of patients with stroke and experimental animals. Also, cells with features of APCs are found in the injured brain tissue after stroke, but the nature of these cells must be better characterized along time to understand their function. Several lines of evidence suggest that brain antigens are presented to $\mathrm{T}$ cells, and, under certain circumpstances involving systemic inflammation, they can elicit autoreactive responses that seem to impair the functional outcome of stroke. Further studies are needed to unravel the actual meaning of these observations. Deciphering complex brain-immune system networks using currently available nextgeneration sequencing, high throughput screening, and bioinformatics should contribute to understand the consequences of the inflammatory and immune responses for the functional outcome of stroke.

Acknowledgments This work was supported by the Spanish Ministries of Economy MINECO (SAF2014-56279-R) and Health (FIS PI12/01437), and the European Community FP7 (FP7-PEOPLE-2013ITN-n ${ }^{\circ} 07962$, supporting M.G.). F.M.M. is supported by the "Red Invictus" of the Instituto de Salud Carlos III (RD12/0014/0011).

\section{References}

1. Ransohoff RM, Engelhardt B. The anatomical and cellular basis of immune surveillance in the central nervous system. Nat Rev Immunol 2012;12:623-635.

2. Gelderblom M, Leypoldt F, Steinbach K, et al. Temporal and spatial dynamics of cerebral immune cell accumulation in stroke. Stroke 2009;40:1849-1857.

3. Gronberg NV, Johansen FF, Kristiansen U, Hasseldam H. Leukocyte infiltration in experimental stroke. J Neuroinflammation 2013;10:115.

4. Iadecola C, Anrather J. The immunology of stroke: from mechanisms to translation. Nat Med 2011;17:796-808.

5. Chamorro Á, Meisel A, Planas AM, Urra X, van de Beek D, Veltkamp R. The immunology of acute stroke. Nat Rev Neurol 2012;8:401-410

6. Kostulas N, Li HL, Xiao BG, Huang YM, Kostulas V, Link H. Dendritic cells are present in ischemic brain after permanent middle cerebral artery occlusion in the rat. Stroke 2002;33:1129-1134.

7. Reichmann G, Schroeter M, Jander S, Fischer HG. Dendritic cells and dendritic-like microglia in focal cortical ischemia of the mouse brain. J Neuroimmunol 2002;129:125-132.

8. Felger JC, Abe T, Kaunzner UW, et al. Brain dendritic cells in ischemic stroke: time course, activation state, and origin. Brain Behav Immun 2010;24:724-737.

9. Pösel C, Uri A, Schulz I, Boltze J, Weise G, Wagner D-C. Flow cytometric characterization of brain dendritic cell subsets after murine stroke. Exp Transl Stroke Med 2014;6:11.

10. Ludewig P, Gallizioli M, Urra X, et al. Dendritic cells in brain diseases. Biochim Biophys Acta 2016;1862:352-367.

11. Schwab JM, Nguyen TD, Meyermann R, Schluesener HJ.Human focal cerebral infarctions induce differential lesional interleukin16 (IL-16) expression confined to infiltrating granulocytes, CD8+ T-lymphocytes and activated microglia/macrophages. J Neuroimmunol 2001;114:232-241.

12. Yilmaz G, Arumugam TV, Stokes KY, Granger DN. Role of T lymphocytes and interferon-gamma in ischemic stroke. Circulation 2006;113:2105-2112.

13. Kleinschnitz C, Schwab N, Kraft P, et al. Early detrimental T-cell effects in experimental cerebral ischemia are neither related to adaptive immunity nor thrombus formation. Blood 2010;115:3835-3842.

14. Gelderblom M, Weymar A, Bernreuther C, et al.Neutralization of the IL-17 axis diminishes neutrophil invasion and protects from ischemic stroke. Blood 2012; 120:3793-3802.

15. Becker KJ, McCarron RM, Ruetzler C, et al. Immunologic tolerance to myelin basic protein decreases stroke size after transient focal cerebral ischemia. Proc Natl Acad Sci U S A 1997;94: 10873-10878.

16. Becker K, Kindrick D, McCarron R, Hallenbeck J, Winn R. Adoptive transfer of myelin basic protein-tolerized splenocytes to naive animals reduces infarct size: a role for lymphocytes in ischemic brain injury? Stroke 2003;34:1809-1815.

17. Chen Y, Ruetzler C, Pandipati S, et al. Mucosal tolerance to Eselectin provides cell-mediated protection against ischemic brain injury. Proc Natl Acad Sci U S A 2003;100:15107-15112.

18. Frenkel D, Huang Z, Maron R, et al. Nasal vaccination with myelin oligodendrocyte glycoprotein reduces stroke size by inducing IL-10-producing CD4+ T cells. J Immunol 2003;171:6549-6555.

19. Gee JM, Kalil A, Thullbery M, Becker KJ. Induction of immunologic tolerance to myelin basic protein prevents central nervous system autoimmunity and improves outcome after stroke. Stroke 2008;39:1575-1582.

20. Ortega SB, Noorbhai I, Poinsatte K, et al. Stroke induces a rapid adaptive autoimmune response to novel neuronal antigens. Discov Med 2015;19:381-392. 
21. Römer C, Engel O, Winek K, et al. Blocking stroke-induced immunodeficiency increases CNS antigen-specific autoreactivity but does not worsen functional outcome after experimental stroke. J Neurosci 2015;35:7777-7794.

22. Planas AM, Gómez-Choco M, Urra X, Gorina R, Caballero M, Chamorro Á. Brain-derived antigens in lymphoid tissue of patients with acute stroke. J Immunol 2012;188:2156-2163.

23. Yang Y, Rosenberg GA. Blood-brain barrier breakdown in acute and chronic cerebrovascular disease. Stroke 2011;42:3323-3328.

24. Weller RO, Djuanda E, Yow HY, Carare RO. Lymphatic drainage of the brain and the pathophysiology of neurological disease. Acta Neuropathol 2009;117:1-14.

25. Iliff JJ, Lee H, Yu M, et al. Brain-wide pathway for waste clearance captured by contrast-enhanced MRI. J Clin Invest 2013;123: 1299-1309.

26. Carare RO, Hawkes CA, Weller RO. Afferent and efferent immunological pathways of the brain. Anatomy, function and failure. Brain Behav Immun 2014;36:9-14.

27. Morris AW, Sharp MM, Albargothy NJ, et al. Vascular basement membranes as pathways for the passage of fluid into and out of the brain. Acta Neuropathol 2016;131:725-736.

28. Tarasoff-Conway JM, Carare RO, Osorio RS, et al. Clearance systems in the brain-implications for Alzheimer diseaser. Nat Rev Neurol 2016;12:248.

29. Cserr HF, Harling-Berg CJ, Knopf PM. Drainage of brain extracellular fluid into blood and deep cervical lymph and its immunological significance. Brain Pathol 1992;2:269-276.

30. Weller RO, Kida S, Zhang ET. Pathways of fluid drainage from the brain-morphological aspects and immunological significance in rat and man. Brain Pathol 1992;2:277-284.

31. Abbott NJ. Evidence for bulk flow of brain interstitial fluid: significance for physiology and pathology. Neurochem Int 2004;45: 545-552.

32. Louveau A, Smirnov I, Keyes TJ, et al. Structural and functional features of central nervous system lymphatic vessels. Nature 2015;523:337-341.

33. Aspelund A, Antila S, Proulx ST, et al. A dural lymphatic vascular system that drains brain interstitial fluid and macromolecules. J Exp Med 2015;212:991-999.

34. Hatterer E, Davoust N, Didier-Bazes M, et al. How to drain without lymphatics? Dendritic cells migrate from the cerebrospinal fluid to the B-cell follicles of cervical lymph nodes. Blood 2006;107:806-812.

35. Steinman RM, Nussenzweig MC. Avoiding horror autotoxicus: the importance of dendritic cells in peripheral $\mathrm{T}$ cell tolerance. Proc Natl Acad Sci U S A 2002;99:351-358.

36. Prass K, Meisel C, Hoflich C, et al. Stroke-induced immunodeficiency promotes spontaneous bacterial infections and is mediated by sympathetic activation reversal by poststroke $\mathrm{T}$ helper cell type 1-like immunostimulation. J Exp Med 2003;198:725-736.

37. Benakis C, Brea D, Caballero S, et al. Commensal microbiota affects ischemic stroke outcome by regulating intestinal $\gamma \delta$ T cells. Nat Med 2016;22:516-523.

38. Dalmau J, Lancaster E, Martinez-Hernandez E, Rosenfeld MR, Balice-Gordon R. Clinical experience and laboratory investigations in patients with anti-NMDAR encephalitis. Lancet Neurol 2011;10:63-74.

39. Zerche M, Weissenborn K, Ott C, et al. Preexisting serum autoantibodies against the NMDAR subunit NR1 modulate evolution of lesion size in acute ischemic stroke. Stroke 2015;46:1180-1186.

40. Dirnagl U, Iadecola C, Moskowitz MA. Pathobiology of ischaemic stroke: an integrated view. Trends Neurosci 1999;22:391-397.

41. Gelderblom M, Sobey CG, Kleinschnitz C, Magnus T. Danger signals in stroke. Ageing Res Rev 2015;24:77-82.

42. Matzinger P. The danger model: a renewed sense of self. Science 2002;296:301-305.
43. Greter M, Heppner FL, Lemos MP, et al. Dendritic cells permit immune invasion of the CNS in an animal model of multiple sclerosis. Nat Med 2005;11:328-334.

44. Prodinger C, Bunse J, Kruger M, et al. CD11c-expressing cells reside in the juxtavascular parenchyma and extend processes into the glia limitans of the mouse nervous system. Acta Neuropathol 2011;121:445-458.

45. Gottfried-Blackmore A, Kaunzner UW, Idoyaga J, Felger JC, McEwen BS, Bulloch K. Acute in vivo exposure to interferongamma enables resident brain dendritic cells to become effective antigen presenting cells. Proc Natl Acad Sci U S A 2009;106: 20918-20923.

46. Reboldi A, Coisne C, Baumjohann D, et al. C-C chemokine receptor 6-regulated entry of TH-17 cells into the CNS through the choroid plexus is required for the initiation of EAE. Nat Immunol 2009; 10:514-523.

47. Dando SJ, Golborne C, Chinnery HR, Ruitenberg MJ, McMenamin PG. A case of mistaken identity: CD11c-eYFP(+) cells in the normal mouse brain parenchyma and neural retina display the phenotype of microglia, not dendritic cells. Glia 2016;64:1331-1349.

48. Savina A, Jancic C, Hugues S, et al. NOX2 controls phagosomal $\mathrm{pH}$ to regulate antigen processing during crosspresentation by dendritic cells. Cell 2006;126:205-218.

49. Guermonprez P, Valladeau J, Zitvogel L, Théry C, Amigorena S. Antigen presentation and $\mathrm{T}$ cell stimulation by dendritic cells. Annu Rev Immunol 2002;20:621-667.

50. Reis e Sousa C. Dendritic cells in a mature age. Nat Rev Immunol 2006;6:476-483.

51. Krummel MF, Davis MM. Dynamics of the immunological synapse: finding, establishing and solidifying a connection. Curr Opin Immunol 2002;14:66-74.

52. Chen L, Flies DB. Molecular mechanisms of T cell co-stimulation and co-inhibition. Nat Rev Immunol 2013;13:227-242.

53. Depoil D, Dustin ML. Force and affinity in ligand discrimination by the TCR. Trends Immunol 2014;35:597-603.

54. Hivroz C, Saitakis M. Biophysical aspects of T lymphocyte activation at the immune synapse. Front Immunol 2016;7:46.

55. Jauch EC, Lindsell C, Broderick J, et al. Association of serial biochemical markers with acute ischemic stroke: the National Institute of Neurological Disorders and Stroke recombinant tissue plasminogen activator Stroke Study. Stroke 2006;37:2508-2513.

56. de Vos AF, van Meurs M, Brok HP, et al. Transfer of central nervous system autoantigens and presentation in secondary lymphoid organs. J Immunol 2002;169:5415-5423.

57. Bartholomaus I, Kawakami N, Odoardi F, et al. Effector T cell interactions with meningeal vascular structures in nascent autoimmune CNS lesions. Nature 2009;462:94-98.

58. Zhang J, Wang Y, Zhu P, Wang X, Lv M, Feng H. siRNAmediated silence of protease-activated receptor- 1 minimizes ischemic injury of cerebral cortex through HSP70 and MAP2. J Neurol Sci 2012;320:6-11.

59. Urra X, Miró F, Chamorro A, Planas AM. Antigen-specific immune reactions to ischemic stroke. Front Cell Neurosci 2014;8:278.

60. Ren X, Akiyoshi K, Grafe MR, et al. Myelin specific cells infiltrate MCAO lesions and exacerbate stroke severity. Metab Brain Dis 2012;27:7-15.

61. Chu HX, Kim HA, Lee S, et al. Immune cell infiltration in malignant middle cerebral artery infarction: comparison with transient cerebral ischemia. J Cereb Blood Flow Metab 2014;34:450-459.

62. Mracsko E, Javidi E, Na SY, Kahn A, Liesz A, Veltkamp R. Leukocyte invasion of the brain after experimental intracerebral hemorrhage in mice. Stroke 2014;45:2107-2114.

63. Liesz A, Suri-Payer E, Veltkamp C, et al. Regulatory T cells are key cerebroprotective immunomodulators in acute experimental stroke. Nat Med 2009;15:192-199. 
64. Stubbe T, Ebner F, Richter D, et al. Regulatory T cells accumulate and proliferate in the ischemic hemisphere for up to 30 days after MCAO. J Cereb Blood Flow Metab 2012;33:37-47.

65. Odoardi F, Sie C, Streyl K, et al. T cells become licensed in the lung to enter the central nervous system. Nature 2012;488:675-679.

66. Rescigno M. Dendritic cells in tolerance induction for the treatment of autoimmune diseases. Eur J Immunol 2010;40:2119-2123.

67. Ganguly D, Haak S, Sisirak V, Reizis B. The role of dendritic cells in autoimmunity. Nat Rev Immunol 2013;13:566-577.

68. Mohammad MG, Tsai VWW, Ruitenberg MJ, et al. Immune cell trafficking from the brain maintains CNS immune tolerance. J Clin Invest 2014;124:1228-1241.

69. Weiner HL. The mucosal milieu creates tolerogenic dendritic cells and T(R)1 and T(H)3 regulatory cells. Nat Immunol 2001;2:671-672.

70. Offner H, Subramanian S, Parker SM, et al. Splenic atrophy in experimental stroke is accompanied by increased regulatory T cells and circulating macrophages. J Immunol 2006;176:6523-6531.

71. Kleinschnitz C, Kraft P, Dreykluft A, et al. Regulatory T cells are strong promoters of acute ischemic stroke in mice by inducing dysfunction of the cerebral microvasculature. Blood 2013;121: 679-691.

72. Schuhmann MK, Kraft P, Stoll G, et al. CD28 superagonistmediated boost of regulatory $\mathrm{T}$ cells increases thromboinflammation and ischemic neurodegeneration during the acute phase of experimental stroke. J Cereb Blood Flow Metab 2015;35:6-10.

73. Na S-YY, Mracsko E, Liesz A, Hünig T, Veltkamp R. Amplification of regulatory $\mathrm{T}$ cells using a CD28 superagonist reduces brain damage after ischemic stroke in mice. Stroke 2015;46:212-220.

74. Liesz A, Hu X, Kleinschnitz C, Offner H. Functional role of regulatory lymphocytes in stroke: facts and controversies. Stroke 2015;46:1422-1430.

75. Li P, Gan Y, Sun B-LL, et al. Adoptive regulatory T-cell therapy protects against cerebral ischemia. Ann Neurol 2013;74:458-471.

76. Li P, Mao L, Zhou G, et al. Adoptive regulatory T-cell therapy preserves systemic immune homeostasis after cerebral ischemia. Stroke 2013;44:35093515.

77. Rostrom B, Link B. Oligoclonal immunoglobulins in cerebrospinal fluid in acute cerebrovascular disease. Neurology 1981;31: 590-596.

78. Pruss H, Iggena D, Baldinger T, et al. Evidence of intrathecal immunoglobulin synthesis in stroke: a cohort study. Arch Neurol 2012;69:714-717.

79. Bornstein NM, Aronovich B, Korczyn AD, Shavit S, Michaelson DM, Chapman J. Antibodies to brain antigens following stroke. Neurology 2001;56:529-530.

80. Dambinova SA, Khounteev GA, Izykenova GA, Zavolokov IG, Ilyukhina AY, Skoromets AA. Blood test detecting autoantibodies to N-methyl-D-aspartate neuroreceptors for evaluation of patients with transient ischemic attack and stroke. Clin Chem 2003;49: 1752-1762.

81. Weissman JD, Khunteev GA, Heath R, Dambinova SA. NR2 antibodies: risk assessment of transient ischemic attack (TIA)/ stroke in patients with history of isolated and multiple cerebrovascular events. J Neurol Sci 2011;300:97102.

82. Hammer C, Stepniak B, Schneider A, et al. Neuropsychiatric disease relevance of circulating anti-NMDA receptor autoantibodies depends on blood-brain barrier integrity. Mol Psychiatry 2014;19: 1143-1149.

83. Dahm L, Ott C, Steiner J, et al. Seroprevalence of autoantibodies against brain antigens in health and disease. Ann Neurol 2014;76: 82-94.

84. Doyle KP, Quach LN, Sole M, et al. B-lymphocyte-mediated delayed cognitive impairment following stroke. J Neurosci 2015;35: 2133-2145.
85. Becker KJ, Tanzi P, Zierath D, Buckwalter MS. Antibodies to myelin basic protein are associated with cognitive decline after stroke. J Neuroimmunol 2016;295:9-11.

86. Kuan AP, Zuckier L, Liao L, Factor SM, Diamond B. Immunoglobulin isotype determines pathogenicity in antibodymediated myocarditis in naive mice. Circ Res 2000;86:281-285.

87. Tuzun E, Zhou L, Baehring JM, Bannykh S, Rosenfeld MR, Dalmau J. Evidence for antibody-mediated pathogenesis in antiNMDAR encephalitis associated with ovarian teratoma. Acta Neuropathol 2009;118:737-743.

88. Kayser MS, Dalmau J. Anti-NMDA receptor encephalitis, autoimmunity, and psychosis. Schizophr Res 2016;176:36-40.

89. Chen PC, Tseng TC, Hsieh JY, Lin HW. Association between stroke and patients with pelvic inflammatory disease: a nationwide population-based study in Taiwan. Stroke 2011;42:2074-2076.

90. Rahman MM, Kopec JA, Cibere J, Goldsmith CH, Anis AH. The relationship between osteoarthritis and cardiovascular disease in a population health survey: a cross-sectional study. BMJ Open 2013;3.

91. Tall AR, Yvan-Charvet L. Cholesterol, inflammation and innate immunity. Nat Rev Immunol 2015;15:104-116.

92. Andersson J, Libby P, Hansson GK. Adaptive immunity and atherosclerosis. Clin Immunol 2010;134:33-46.

93. Zhou X, Nicoletti A, Elhage R, Hansson GK. Transfer of CD4(+) $T$ cells aggravates atherosclerosis in immunodeficient apolipoprotein E knockout mice. Circulation 2000;102:2919-2922.

94. Reardon CA, Blachowicz L, White T, et al. Effect of immune deficiency on lipoproteins and atherosclerosis in male apolipoprotein E-deficient mice. Arterioscler Thromb Vasc Biol 2001;21: 1011-1016.

95. Ryu H, Chung Y. Regulation of IL-17 in atherosclerosis and related autoimmunity. Cytokine 2015;74:219-227.

96. Reynolds CM, McGillicuddy FC, Harford KA, Finucane OM, Mills KH, Roche HM. Dietary saturated fatty acids prime the NLRP3 inflammasome via TLR4 in dendritic cells-implications for diet-induced insulin resistance. Mol Nutr Food Res 2012;56: 1212-1222.

97. Erbel C, Dengler TJ, Wangler S, et al. Expression of IL-17A in human atherosclerotic lesions is associated with increased inflammation and plaque vulnerability. Basic Res Cardiol 2011;106:125-134.

98. Lim H, Kim YU, Sun H, et al. Proatherogenic conditions promote autoimmune $\mathrm{T}$ helper 17 cell responses in vivo. Immunity 2014:40:153-165.

99. Peters MJ, Symmons DP, McCarey D, et al. EULAR evidencebased recommendations for cardiovascular risk management in patients with rheumatoid arthritis and other forms of inflammatory arthritis. Ann Rheum Dis 2010;69:325-331.

100. Wiseman SJ, Ralston SH, Wardlaw JM. Cerebrovascular disease in rheumatic diseases: a systematic review and meta-analysis. Stroke 2016;47:943-950.

101. Portegies ML, Lahousse L, Joos GF, et al. Chronic Obstructive Pulmonary Disease and the Risk of Stroke. The Rotterdam Study. Am J Respir Crit Care Med 2016;193:251-258.

102. Denes A, Thornton P, Rothwell NJ, Allan SM. Inflammation and brain injury: acute cerebral ischaemia, peripheral and central inflammation. Brain Behav Immun 2010;24:708-723.

103. Sherer Y, Shoenfeld Y. Mechanisms of disease: atherosclerosis in autoimmune diseases. Nat Clin Pract Rheumatol 2006;2:99-106.

104. Boehme AK, Kapoor N, Albright KC, et al. Systemic inflammatory response syndrome in tissue-type plasminogen activatortreated patients is associated with worse short-term functional outcome. Stroke 2013;44:2321-2323.

105. Murray KN, Girard S, Holmes WM, et al. Systemic inflammation impairs tissue reperfusion through endothelin-dependent mechanisms in cerebral ischemia. Stroke 2014;45:3412-3419.

106. Denes A, Ferenczi S, Kovacs KJ. Systemic inflammatory challenges compromise survival after experimental stroke via 
augmenting brain inflammation, blood- brain barrier damage and brain oedema independently of infarct size. J Neuroinflammation 2011;8:164

107. McColl BW, Rothwell NJ, Allan SM. Systemic inflammation alters the kinetics of cerebrovascular tight junction disruption after experimental stroke in mice. J Neurosci 2008;28:9451-9462.

108. Pérez-de Puig I, Miró F, Salas-Perdomo A, et al. IL-10 deficiency exacerbates the brain inflammatory response to permanent ischemia without preventing resolution of the lesion. J Cereb Blood Flow Metab 2013;33:1955-1966.

109. Goverman J. Autoimmune T cell responses in the central nervous system. Nat Rev Immunol 2009;9:393-407.

110. Ransohoff RM, Kivisakk P, Kidd G. Three or more routes for leukocyte migration into the central nervous system. Nat Rev Immunol 2003;3:569-581.

111. Lindsberg PJ, Grau AJ. Inflammation and infections as risk factors for ischemic stroke. Stroke 2003;34:2518-2532.

112. Emsley HC, Hopkins SJ. Acute ischaemic stroke and infection: recent and emerging concepts. Lancet Neurol 2008;7:341-353.

113. Elkind MS, Carty CL, O'Meara ES, et al. Hospitalization for infection and risk of acute ischemic stroke: the Cardiovascular Health Study. Stroke 2011;42:1851-1856.

114. Donze JD, Ridker PM, Finlayson SR, Bates DW. Impact of sepsis on risk of postoperative arterial and venous thromboses: large prospective cohort study. BMJ 2014;349:g5334.

115. Zeller JA, Lenz A, Eschenfelder CC, Zunker P, Deuschl G. Platelet-leukocyte interaction and platelet activation in acute stroke with and without preceding infection. Arterioscler Thromb Vasc Biol 2005;25:1519-1523.

116. Heikinheimo T, Broman J, Haapaniemi E, Kaste M, Tatlisumak T, Putaala J. Preceding and poststroke infections in young adults with first-ever ischemic stroke: effect on short-term and long-term outcomes. Stroke 2013;44:3331-3337.

117. Kumar S, Selim MH, Caplan LR. Medical complications after stroke. Lancet Neurol 2010;9:105-118.

118. Westendorp WF, Nederkoorn PJ, Vermeij JD, Dijkgraaf MG, van de Beek D. Post-stroke infection: a systematic review and metaanalysis. BMC Neurol 2011;11:110.

119. Chamorro A, Urra X, Planas AM. Infection after acute ischemic stroke: a manifestation of brain-induced immunodepression. Stroke 2007;38:1097-1103.

120. Banchereau J, Briere F, Caux C, et al. Immunobiology of dendritic cells. Annu Rev Immunol 2000;18:767-811.

121. Getts DR, Chastain EM, Terry RL, Miller SD. Virus infection, antiviral immunity, and autoimmunity. Immunol Rev 2013;255:197-209.

122. Berer K, Krishnamoorthy G. Microbial view of central nervous system autoimmunity. FEBS Lett 2014;588:4207-4213.

123. Becker KJ, Kindrick DL, Lester MP, Shea C, Ye Z-CC. Sensitization to brain antigens after stroke is augmented by lipopolysaccharide. J Cereb Blood Flow Metab 2005;25:1634-1644.

124. Becker KJ, Kalil AJ, Tanzi P, et al. Autoimmune responses to the brain after stroke are associated with worse outcome. Stroke 2011;42:2763-2769.

125. Zierath D, Thullbery M, Hadwin J, et al. CNS immune responses following experimental stroke. Neurocrit Care 2010;12:274-284.

126. Vargas M, Horcajada JP, Obach V, et al. Clinical consequences of infection in patients with acute stroke: is it prime time for further antibiotic trials? Stroke 2006;37:461-465.

127. Hug A, Dalpke A, Wieczorek N, et al. Infarct volume is a major determiner of post-stroke immune cell function and susceptibility to infection. Stroke 2009;40:3226-3232.

128. Urra X, Chamorro A. Stroke-induced immunodepression is a marker of severe brain damage. Stroke 2010;41:e110.
129. Haeusler KG, Schmidt WU, Fohring F, et al. Cellular immunodepression preceding infectious complications after acute ischemic stroke in humans. Cerebrovasc Dis 2008;25:50-58.

130. Klehmet J, Harms H, Richter M, et al. Stroke-induced immunodepression and post-stroke infections: lessons from the preventive antibacterial therapy in stroke trial. Neuroscience 2009;158:1184-1193.

131. Urra X, Cervera A, Villamor N, Planas AM, Chamorro A. Harms and benefits of lymphocyte subpopulations in patients with acute stroke. Neuroscience 2009;158:1174-1183.

132. Vogelgesang A, Grunwald U, Langner S, et al. Analysis of lymphocyte subsets in patients with stroke and their influence on infection after stroke. Stroke 2008;39:237-241.

133. Wong CH, Jenne CN, Lee WY, Leger C, Kubes P. Functional innervation of hepatic iNKT cells is immunosuppressive following stroke. Science 2011;334:101-105.

134. Trakhtenberg EF, Goldberg JL. Immunology. Neuroimmune communication. Science 2011;334:47-48.

135. Seiffert K, Hosoi J, Torii H, et al. Catecholamines inhibit the antigen-presenting capability of epidermal Langerhans cells. J Immunol 2002;168:6128-6135.

136. Maestroni GJ, Mazzola P. Langerhans cells beta 2-adrenoceptors: role in migration, cytokine production, Th priming and contact hypersensitivity. J Neuroimmunol 2003;144:91-99.

137. Herve J, Dubreil L, Tardif V, et al. beta2-Adrenoreceptor agonist inhibits antigen cross-presentation by dendritic cells. J Immunol 2013;190:3163-3171.

138. de Kruyff RH, Fang Y, Umetsu DT. Corticosteroids enhance the capacity of macrophages to induce Th2 cytokine synthesis in CD4+ lymphocytes by inhibiting IL-12 production. J Immunol 1998; 160:2231-2237.

139. de Jong EC, Vieira PL, Kalinski P, Kapsenberg ML. Corticosteroids inhibit the production of inflammatory mediators in immature monocyte-derived DC and induce the development of tolerogenic DC3. J Leukoc Biol 1999;66:201-204.

140. Varga G, Ehrchen J, Brockhausen A, et al. Immune suppression via glucocorticoid-stimulated monocytes: a novel mechanism to cope with inflammation. J Immunol 2014;193:1090-1099.

141. Steinbrink K, Graulich E, Kubsch S, Knop J, Enk AH. CD4(+) and CD8(+) anergic T cells induced by interleukin-10-treated human dendritic cells display antigen-specific suppressor activity. Blood 2002;99:2468-2476.

142. Radjavi A, Smirnov I, Kipnis J. Brain antigen-reactive CD4+ T cells are sufficient to support learning behavior in mice with limited T cell repertoire. Brain Behav Immun 2014;35:58-63.

143. Gee JM, Kalil A, Shea C, Becker KJ. Lymphocytes: potential mediators of postischemic injury and neuroprotection. Stroke 2007;38:783-788.

144. Schwartz M, Raposo C. Protective autoimmunity: a unifying model for the immune network involved in CNS repair. Neuroscientist 2014;20:343-358

145. Liu J, Zhang C, Tao W, Liu M. Systematic review and metaanalysis of the efficacy of sphingosine-1-phosphate (S1P) receptor agonist FTY720 (fingolimod) in animal models of stroke. Int J Neurosci 2013;123:163-169.

146. Fu Y, Zhang N, Ren L, et al. Impact of an immune modulator fingolimod on acute ischemic stroke. Proc Natl Acad Sci U S A 2014;111:18315-18320.

147. Zhu Z, Fu Y, Tian D, et al. Combination of the immune modulator fingolimod with alteplase in acute ischemic stroke: a pilot trial. Circulation 2015;132:1104-1112

148. Llovera G, Hofmann K, Roth S, et al. Results of a preclinical randomized controlled multicenter trial (pRCT): Anti-CD49d treatment for acute brain ischemia. Sci Transl Med 2015;7: 299ra121 